\title{
BCMA x CD3 T-cell Engaging Antibody CC- 93269
}

National Cancer Institute

\section{Source}

National Cancer Institute. BCMA x CD3 T-cell Engaging Antibody CC-93269. NCI

Thesaurus. Code C156052.

A bispecific T-cell engager (BITE) antibody composed of two single-chain variable fragments (scFv), one directed against the tumor-associated antigen (TAA) human B-cell maturation antigen (BCMA; TNFRSF17), and one that is directed against the CD3 antigen found on T-lymphocytes, with potential immunostimulating and antineoplastic activities. Upon administration of anti-BCMA/CD3 T-cell eng aging antibody CC-93269, this bispecific antibody binds to both CD3 on cytotoxic T-lymphocytes (CT Ls) and BCMA found on BCMA-expressing tumor cells. This activates and redirects CT LS to BCMAexpressing tumor cells, which results in the CT L-mediated death of BCMA-expressing tumor cells. BCMA, a member of the tumor necrosis factor receptor superfamily that is specifically overexpressed on malignant plasma cells, plays a key role in promoting plasma cell survival. 